\title{
Location problems in renewable sensor networks with wireless energy transfer*
}

\author{
Denis Migov
}

\begin{abstract}
The area of wireless sensor networks (WSNs) has recently received a lot of attention. Nevertheless, the major problem is the finite electrical batteries in sensors, which prevents the wide use of WSNs. However, technologies of the wireless energy transfer may be applied to solve energy problems in WSNs with the use of wireless energy chargers. In this study we offer the techniques of finding the points, which are most suitable for a simultaneous omnidirectional wireless charge of a group of sensors. The wireless chargers in question can be both mobile or static. The stated problems are quite similar to the problems of $p$-center and $p$-median location in a graph and may be solved by appropriate methods. The approach proposed may significantly decrease a path of a mobile charger when traversing places for recharging sensors. For the WSNs with static wireless chargers these optimal points are useful for the chargers deployment.
\end{abstract}

\section{Introduction}

The wireless sensor networks are gaining more importance due to many applications [1-4]. As a rule, a WSN consists of a generous amount of micro sensors, which monitor required index and can establish a wireless communication with each other for delivering gathered data. These data may be temperature, pressure, speed, etc.

At the same time, WSNs experience difficulties due to finite electrical batteries in sensors. So, most of wireless sensor networks can keep functioning just for a constrained period. Nowadays, engineers and researchers are trying to find green energy sources, which make possible to recharge batteries. This approach, known as energy harvesting, can provide autonomous functioning of WSNs, without necessity for battery replacement. We may classify the energy sources used into three kinds $[1,2]$ : thermal energy, radiant energy, and mechanical energy.

Another approach to overcome energy problems of WSNs is the wireless energy transfer [5-7], which gives an alternative opportunity to prolong the wireless sensor network lifetime [8-11]. As shown in [9], there are three ways of wireless energy transfer: inductive coupling, electromagnetic radiation and magnetic resonant coupling. Among them, the technology of magnetic

\footnotetext{
* Supported by the Basic Research Program No. 1.31 of the Presidium of the Russian Academy of Sciences.
} 
resonant coupling is most useful for applications in WSNs. This technology, developed by Kurs et al. [6], is based on the principle of resonant coupling. In accord with this technology, energy can be transferred from a source coil to a receiver coil which are operating at the same resonance frequency. The efficiency of energy transfer depends on the size of effective area of the receiver coil in the magnetic field of the transmitter coil.

Further, Kurs et al. [7] developed an enhanced magnetic resonant coupling technology that allows charging multiple devices simultaneously. The efficiency is high within several-meter range. Moreover, there are no requirements of a line of sight and appropriate weather conditions.

The above mentioned technologies may be applied for solving the energy problems in WSNs with the use of wireless energy chargers. One approach is using mobile energy chargers for charging sensors $[8,12-14]$. There are two ways of using this technology. The first one is the so-called offline scenarios $[8,13,14]$ when nodes are charged in a periodic and deterministic manner. The second way of utilization of mobile energy chargers is the on-demand energy replenishment in WSNs [12].

The static wireless energy chargers utilization in WSNs has been subject to research, too [15]. The authors [15] study the WSNs with wireless static chargers equipped with 3D-beamforming directional antennas. It is assumed that chargers are deployed at grid points at a fixed height, and the charging space of a charger equipped with a directional antenna is simulated by a cone. The authors solve the optimization problem of how to deploy as few as possible chargers in a WSN to cover all sensor nodes to make the network sustainable. Two greedy algorithms for solving this optimization problem were proposed.

In this study, we offer some optimization techniques for WSNs with wireless chargers which can be both mobile or static. More specifically, we state problems the search for the points which are most suitable for the simultaneous omnidirectional wireless charging of a group of sensors. These problems are quite similar to those of $p$-center and $p$-median location in a graph and may be solved by appropriate methods.

For the WSNs with mobile chargers, these optimal points should be visited by chargers. This approach may significantly reduce the path of a mobile charger when traversing places for recharging sensors. Further, the optimal path planning for mobile chargers in order to reduce the time of bypass can be performed by one of the known methods [12-14]. For WSNs with static wireless chargers these optimal points are useful for the chargers deployment. 


\section{The basic definitions and notations}

Let us assume that a wireless sensor network is distributed on some twodimensional bounded plane area $\Omega$. Also, we suppose that due to the terrain features, certain subareas are prohibited for visiting by mobile wireless chargers and for placing a static wireless charger on them. We denote the union of these subareas as $P$.

We denote a set of sensors as $S$. A geometrical distance between the two objects $x$ and $y$ on $\Omega$ is denoted as $d(x, y)$. As such objects, we consider sensors and wireless chargers both mobile and static.

We denote as $R_{\mathrm{WCD}}$ a maximum distance at which the wireless power transfer can be performed. That is, the wireless charger WC can recharge a sensor $s$ within a reasonable time iff $d(\mathrm{WC}, s) \leq R_{\mathrm{WCD}}$. Otherwise, the wireless charging is completely ineffective.

\section{A location problem for WSNs with wireless chargers}

In this section, we study the WSNs with wireless energy chargers (WC), which can be mobile (MWC) or static (SWC). One of the primary problems for the WSNs with MWC is the optimal path planning for mobile chargers for reducing the bypass time. With the use of the enhanced magnetic resonant coupling technology [7] it is possible to wireless charge multiple devices at the same time. Thus, MWC may travel inside WSN and visit the points which are most suitable for the simultaneous wireless charging of a group of sensors. In case of static chargers, SWCs may be placed at these points. So, we arrive at the problem of finding a sufficient amount of these points depending on the effective wireless charging distance and their locations. Having these optimal points, we can further solve the path planning problem by one of the existing methods. For example, we may use methods based on finding the shortest Hamiltonian cycle $[8,9]$, the optimal offline path planning $[13,14]$, the on-demand charging method [12], and others methods.

The approach proposed may significantly reduce the mobile charger path when traversing places for recharging sensors. For instance, when using the methods from $[8,9]$, the Hamiltonian cycle is sought for in a graph of a smaller dimension than the initial one. Although the on-demand charging method [12] originally operates with direct contact - based energy transferring technologies, it may also be used for the on-demand wireless charging with minor changes. Instead of visiting each sensor for the direct-contact energy transfer, MEC may visit the above-mentioned points which are most suitable for the simultaneous wireless charging of a group of sensors. Thus, a few sensors may be charged per one request therefore requests the total number of requests is reduces. For the offline path planning methods [13,14], the application of the approach proposed is exactly the same: visiting lo- 
cations which are suitable for the simultaneous wireless charging of a few sensors instead of visiting each sensor separately.

We formulate the location problem of points for visiting by $\mathrm{WC}$ as follows: find the subset $L \subseteq \Omega \backslash P$ with a minimum amount of elements such that

$$
\forall s \in S \quad \exists l \in L: d(s, l) \leq R_{\mathrm{WCD}} .
$$

There are two ways for solving this problem. The first one is the search among points of the continuous set $\Omega \backslash P$. For this purpose methods for the area coverage problem in sensor networks may be useful $[18,19]$.

The second approach is the search among variants from a certain discrete subset $V \subseteq \Omega \backslash P$. The subset $V$ may be formed by various ways. We propose to form it by dividing $\Omega$ into squares of the same size $h$, so we obtain a discrete coordinate grid (the figure). Points of grid lines intersections may be taken as elements of the auxiliary set $W$. The set $V$ is obtained from the set $W$ by elimination of elements from the set $P: V=W \backslash P$.

Let us define the graph $G=(S \cup V, E)$, where $S \cup V$ is a set of vertices and $E$ is a set of edges. An edge between two vertices exist iff one of vertices is from $V$, another is from $S$, and the distance between them is shorter or equal to $R_{\mathrm{WCD}}$.

Thus, the formulated problem is quite similar to that of $p$-center location in the graph $G$. This well-known graph problem is how to locate $p$ centers (service nodes) at graph vertices (served nodes) in order to reduce a distance from the most remote vertice to the nearest center. However, there are certain differences between the $p$-center location problem and problem (1). First, we initially do not know the exact value of $p$. Second, centers should be placed at the nodes from $V$, while the nodes from $S$ are considered as the ones being served. Third, the distance between any sensor and the nearest point for a visit by WC should be shorter or equal to $R_{\mathrm{WCD}}$.

However, despite these differences, known methods for solving the $p$-center problem $[16,17]$ with minor changes can be successfully applied for solving the stated problem (1). Although the exact value of points for visiting by MEC is initially unknown, problem (1) in the discrete case has obviously a decision since condition (1) is satisfied if we assume that $h=R_{\mathrm{WCD}}$.

\section{Reducing energy losses for wireless energy transfer}

It is possible to locate points for the simultaneous wireless charging of sensors more effectively than locations in accordance with the solution of problem (1). The energy losses during the wireless energy transfer grow very fast with increasing the charging distance, even it is shorter than $R_{\mathrm{WCD}}$ value. Hence, we may locate these points in such a way that the sum of distances from each sensor to the nearset point is minimal. Using these points for the simultaneous wireless charging of sensors, we greatly reduce the energy 
transmission losses, the time of wireless charging and the total amount of mobile charger visits to each point. The function of absolute losses energy during the energy transmission at a distance $d$ has the following form:

$$
E_{\mathrm{los}}(d)=E_{\text {sent }}(d)-E_{\text {recieved }}(d) .
$$

The value $E_{\text {recieved }}(d)$ can take different forms depending on many factors. The crude approximation of this function is

$$
E_{\text {recieved }}(d)=\frac{E_{\text {sent }}(d)}{d^{\alpha}}, \quad \alpha>1
$$

If $d>R_{\mathrm{WCD}}, E_{\text {recieved }}(d)$ is close to zero, so we assume $E_{\text {recieved }}(d)=0$ for $d>R_{\mathrm{WCD}}$. Thus, in order to reduce the energy transmission losses, we must minimize the following function:

$$
\sum_{s \in S} E_{\mathrm{los}}(d(s, \text { nearest } \mathrm{WC}))
$$

For solving this problem we may define a discrete coordinate grid and obtain the graph $G=(S \cup V, E)$, like we did in the previous section (see the figure). Thus, the formulated problem is quite similar to the problem of $p$-median location in the graph $G$. This well-known graph problem is how to locate $p$ medians (service nodes) at graph vertices (served nodes) in order to reduce the sum of distances from each vertice to the nearest center. Thus, known methods for solving the $p$-median problem $[16,17]$ with minor changes can successfully be applied for solving the stated problem (4) in a discrete case.

\section{Conclusion}

In this paper, we have presented optimization methods for the WSNs with wireless chargers which can be both mobile or static. We have formulated the problems of the search for the points which are most suitable for the simultaneous omnidirectional wireless charging of a group of sensors. These problems are quite similar to the problems of $p$-center and $p$-median location in a graph and may be solved by appropriate methods.

For the WSNs with mobile chargers, these optimal points should be visited by chargers. This approach may significantly reduce the path of a mobile charger when traversing the places for recharging sensors. Further, the optimal path planning for mobile chargers for reducing the time of bypass can be performed by one of the known methods [12-14]. For the WSNs with static wireless chargers these optimal points are useful for the chargers deployment. 


\section{References}

[1] Seah W., Tan Y.K. Review of energy harvesting technologies for sustainable wireless sensor network. Sustainable wireless sensor networks // Tech. Publishing, Janeza Trdine 9, 51000 Rijeka, Croatia, Dec. 2010; Y.K. Tan, Ed.P. 15-43.

[2] Zungeru A.M., Ang L.-M., Prabaharan S.R.S., Seng K.P. Radio frequency energy harvesting and management for wireless sensor networks // Green Mobile Devices and Networks: Energy Optimization and Scavenging Techniques.2012.- P. 341-368.

[3] Migov D., Shakhov V. Reliability of ad hoc networks with imperfect nodes // Proc. 7th Int. Workshop, MACOM 2014, Halmstad, Sweden, August 27-28, 2014.-- Springer, 2014.- P. 49-58.-(LNCS; 8715).

[4] Shakhov V., Migov D., Rodionov A. Operation strategy for energy harvesting wireless sensor networks // Proc. 9th Int. Conf. on Ubiquitous Information Management and Communication (ACM IMCOM 2015), Bali, Indonesia, 2015.--New York: ACM, 2015.-Article No. 12, doi:10.1145/2701126.2701216.

[5] Tesla N. Apparatus for Transmitting Electrical Energy.- US Patent number 1,119,732, issued in Dec. 1914.

[6] Kurs A., Karalis A., Moffatt R., et al. Wireless power transfer via strongly coupled magnetic resonances // Science.--July, 2007. - Vol. 317, No. 5834.P. 83-86.

[7] Kurs A., Moffatt R., Soljacic M. Simultaneous mid-range power transfer to multiple devices // Appl. Phys. Lett. - Jan., 2010. - Vol. 96. - P. 044102-1044102-3.

[8] Xie L., Shi Y., Hou Y.T., Sherali H.D. Making sensor networks immortal: An energy-renewal approach with wireless power transfer // IEEE/ACM Trans. on Networking. - Dec., 2012.-- Vol. 20, No. 6.- P. 1748-1761.

[9] Xie L., Shi Y., Hou Y.T., Lou A. Wireless power transfer and applications to sensor networks // IEEE Wireless Communications. - 2013. - Vol. 20, No. 4.P. $140-145$.

[10] Peng Y., Li Z., Zhang W., Qiao D. Prolonging sensor network lifetime through wireless charging // RTSS'10, 2010.-IEEE, 2010.-P. 129-139.

[11] Chiu T.Ch., Shih Y.Y., Pang A.Ch., et al. Mobility-aware charger deployment for wireless rechargeable sensor networks // APNOMS, 2012.--IEEE, 2012.P. 1-7.

[12] He L., Gu Y., Pan J., Zhu T. On-demand charging in wireless sensor networks: Theories and applications // MASS'13, 2013. - IEEE, 2013. - P. 28-36.

[13] Fu L., Cheng P., Gu Y., et al. Minimizing charging delay in wireless rechargeable sensor networks // INFOCOM'13, 2013. - IEEE, 2013. - P. 2922-2930. 
[14] Guo S., Wang C., Yuan Y. Mobile data gathering with wireless energy replenishment in rechargeable sensor networks // INFOCOM'13, 2013. - IEEE, 2013. - P.1932-1940.

[15] Liao J.H., Jiang J.R. Wireless charger deployment optimization for wireless rechargeable sensor networks // I Proc. of the 7th International Conference on Ubi-Media Computing (UMEDIA 2014), 2014.--IEEE, 2014.-- P. 160-164.

[16] Hakimi S.L. Optimum locations of switching centers and the absolute centers and medians of a graph // Operations Research. - 1964. - Vol. 12. - No. 3.P. $450-459$.

[17] Christofides N. Graph Theory. An Algorithmic Approach.-Academic Press Inc., 1975.

[18] Zalyubovskiy V., Erzin A., Astrakov S., Choo H. Energy-efficient area coverage by sensors with adjustable ranges // MDPI Sensors.-2009.--Vol. 9.-- P. 24462460 .

[19] Nguyen N.D., Zalyubovskiy V., Ha M.T., et al. Energy-efficient models for coverage problem in sensor networks with adjustable ranges // Ad hoc Sensor Networks. - 2012. - No. 16. - P. 1-28. 
